\title{
Sum-Rate Maximization and Energy-Cost Minimization for Renewable Energy Empowered Base-Stations using Zero-Forcing Beamforming
}

\author{
Yung-Shun Wang*, Y.-W. Peter Hong ${ }^{\dagger}$ and Wen-Tsuen Chen* \\ ${ }^{*}$ Institute of Information Science, Academia Sinica Taipei 11529, Taiwan. \\ ${ }^{\dagger}$ Institute of Communications Engineering, National Tsing Hua University, Hsinchu 30013, Taiwan. \\ Email: yongshanw@iis.sinica.edu.tw, ywhong@ee.nthu.edu.tw,wtchen@iis.sinica.edu.tw
}

\begin{abstract}
Zero-forcing (ZF) beamforming is a practical linear transmission scheme that eliminates inter-user interference in the downlink of a multiuser multiple-input single-output (MISO) wireless system. By considering base-stations (BSs) that are supported by renewable energy, this work examines offline and online $\mathrm{ZF}$ beamforming designs based on two different objectives, namely, sum-rate maximization and energy-cost minimization. For offline policies, the channel states and the energy arrivals are assumed to be known a priori for all time instants whereas, in the online policies, only causal information is available. The designs are subject to energy causality and energy storage constraints, i.e., the constraint that energy cannot be used before it arrives and the constraint that the stored energy cannot exceed the maximum battery storage capacity. In the sum-rate maximization problem, the base-station is assumed to be supported only by renewable energy and the goal is to maximize the sum rate over all users by a predetermined deadline. The optimization of the $\mathrm{ZF}$ beamforming direction and power allocation can be decoupled, and the solutions can be found exactly. In the energycost minimization problem, the base-station is assumed to be supported by both renewable and power-grid energy, and the goal is to minimize the cost of purchasing grid energy subject to quality-of-service constraints at the users. The problem can be formulated as a convex optimization problem and can be solved efficiently using off-the-shelf solvers. Offline solutions are first obtained and the intuitions gained from their results are used to derive effective online policies. The effectiveness of the proposed policies are demonstrated through computer simulations.
\end{abstract}

\section{INTRODUCTION}

Small cell networks [1] have emerged as a promising solution for increasing wireless data-rate and for reducing energy and environmental costs [2]. The shorter transmission distance allows base-stations (BSs) to operate under lower power and enables the use of renewable energy, which is often limited over time. However, the uncertainty of renewable energy arrivals and the limitation on the battery storage capacity pose additional constraints to the downlink transmission and, thus, new beamforming and power control policies must be devised for efficient energy usage and for achieving quality-of-service (QoS) guarantee under time-varying energy constraints.

Downlink beamforming and power control policies have been studied extensively in the literature based on different design criterion, e.g., zero-forcing (ZF) [3]-[6], minimum mean-square error [7]-[9], sum-rate maximization [10]-[12], minimum signal-to-interference-plus-noise ratio (SINR) max- imization [13]-[15], and power minimization [16], [17] etc. In particular, $\mathrm{ZF}$ beamforming [3]-[6] is considered as one of the most popular and practical multiuser beamforming schemes due to its simplicity and ability to eliminate interuser interference in the downlink of a multiuser multiple-input single-output (MISO) wireless system. In fact, this scheme is known to achieve the optimal degrees-of-freedom performance [4] and asymptotically optimal sum-rate performance as the number of users goes to infinity [5]. These techniques have been extended to multiple-input multiple-output (MIMO) broadcast channels in [6] and to multi-cell networks in [18].

The main objective of this work is devise multiuser downlink ZF beamforming and power control policies for efficient usage of renewable energy at a small cell BS. The BS is equipped with multiple antennas and is supported by renewable energy through a rechargeable battery. The policies are derived based on two optimizing criterion: the maximum sumrate criterion and the minimum energy-cost criterion. In the sum-rate maximization problem, the BS is assumed to be supported only by renewable energy and the goal is to maximize the sum-rate of all users and time instants by a deadline. In the energy-cost minimization problem, the BS is assumed to be supported by both renewable energy and grid energy, and the goal is to minimize the total cost of purchasing grid energy over time. Due to the usage of renewable energy, the designs are subject to energy causality and battery storage constraints, i.e., the constraint that energy cannot be used before it arrives and the constraint that the stored energy cannot exceed the maximum storage capacity of the rechargeable battery. First, optimal offline policies are derived based on non-causal knowledge of the energy arrivals and channel coefficients over time. Then, online policies are proposed based on intuitions gained from the offline solutions. In the offline sum-rate maximization problem, the optimization over the beamforming direction and power allocation can be decoupled, and can be solved exactly. This solution inspires an online directional water-filling policy, similar to that proposed in [19] and [20]. In the offline energycost minimization problem, the problem can be formulated as a convex optimization problem and can be efficiently solved using off-the-shelf solvers. The effectiveness of the proposed schemes are demonstrated through computer simulations.

Transmission policies for renewable energy empowered (or 
energy harvesting) devices were studied recently in [19]-[23]. In [19], offline and online power control policies were derived for a single-input single-output (SISO) point-to-point channel. The directional water-filling algorithm was proposed and shown to be optimal in terms of maximizing the throughput by a deadline subject to energy causality and battery storage constraints. The relation between the throughput maximization problem and the problem of minimizing the transmission completion time for a given amount of data was explored in [21]. The latter problem was then extended to the $K$ user AWGN broadcast channel in [22]. In [23], the authors considered random data arrivals and discussed a grid power minimization problem. For the case that all the data is ready before transmission, the problem is shown to be a dual problem of the throughput maximization problem. Different from these works, we consider the multiuser downlink beamforming scenario with a multi-antenna BS. The policies are derived for sum-rate maximization and energy-cost minimization. The first problem was partially studied in [20] without design of the ZF beamformer. The second problem, however, has not been explored before in the literature.

The rest of this paper is organized as follows. Section II introduces the system model and problem formulations. Sections III and IV derive the optimal solutions for the sumrate maximization and the energy-cost minimization problems, respectively. Section V develops heuristic online policies based on the offline solutions. Section VI provides numerical simulations to demonstrate the effectiveness of the proposed schemes. Finally, Section VII concludes the paper.

\section{System Model And Problem Formulation}

Let us consider a downlink wireless cellular system with a multi-antenna BS transmitting to $K$ single-antenna users, as illustrated in Fig. 1. The BS is equipped with $M$ antennas and is supported by renewable energy through a rechargeable battery. Here, we consider a time-slotted system and assume that the channels remain constant within each time slot. All $K$ users are served simultaneously by the BS in each time slot. In this case, it is necessary to have $M \geq K$ in order to completely eliminate inter-user interference.

Let $s_{i}[t]$ be the signal intended for user $i$ in time slot $t$ and let $\mathbf{w}_{i}[t]$ be the associated $M \times 1$ beamforming vector. Assume that $s_{i}[t]$ is Gaussian with zero mean and unit variance, and is independent and identically distributed (i.i.d.) for all $i$ and $t$. The signal transmitted by the BS in time slot $t$ can then be expressed as $\mathbf{x}[t]=\sum_{i=1}^{K} \mathbf{w}_{i}[t] s_{i}[t]$, whose power is given by $\mathrm{E}\left[\|\mathbf{x}[t]\|^{2}\right]=\sum_{i=1}^{K}\left\|\mathbf{w}_{i}[t]\right\|^{2}$. The received signal at user $i$ is given by

$$
y_{i}[t]=\mathbf{h}_{i}[t]^{H} \mathbf{w}_{i}[t] s_{i}[t]+\mathbf{h}_{i}[t]^{H} \sum_{j \neq i} \mathbf{w}_{j}[t] s_{j}[t]+n_{i}[t],
$$

where $\mathbf{h}_{i}[t]$ is the $M \times 1$ channel vector between the BS and user $i$ and $n_{i}[t]$ is the additive white Gaussian noise (AWGN) with zero mean and variance $\sigma_{n}^{2}$, i.e., $n_{i}[t] \sim \mathcal{C N}\left(0, \sigma_{n}^{2}\right)$. The first term in (1) is the signal intended for user $i$ while the second term contains the interference from other signals.

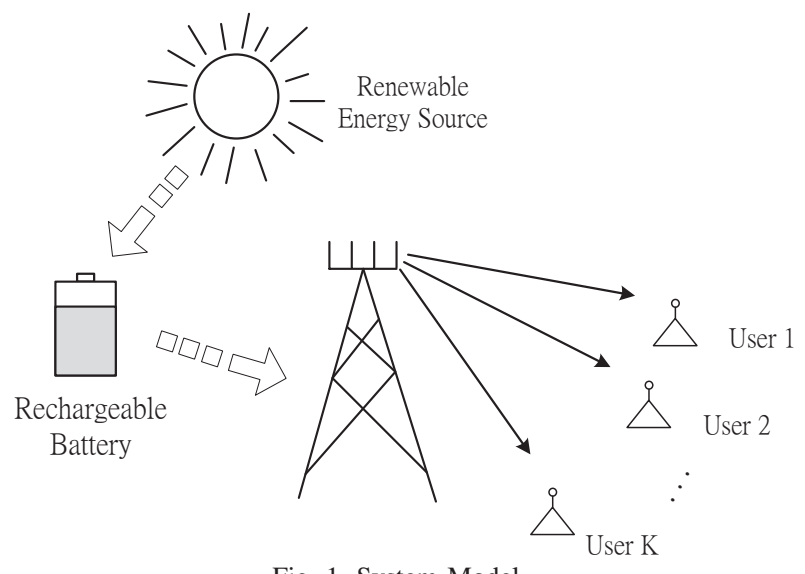

Fig. 1. System Model

To eliminate inter-user interference, the beamforming vectors $\mathbf{w}_{j}[t]$, for $j=1, \ldots, K$, are chosen such that

$$
\mathbf{h}_{i}[t]^{H} \mathbf{w}_{j}[t]=0, \forall i \neq j .
$$

The signal-to-noise ratio (SNR) at user $i$ is given by $\mathrm{SNR}_{i}[t]=$ $\left|\mathbf{h}_{i}[t]^{H} \mathbf{w}_{i}[t]\right|^{2} / \sigma_{n}^{2}$ and, thus, the rate achieved by user $i$ in the $t$-th time slot is $R_{i}[t]=\log \left(1+\mathrm{SNR}_{i}[t]\right)$. Therefore, the sum rate achieved by the deadline $T$ can be expressed as

$$
\sum_{t=1}^{T} \sum_{i=1}^{K} \log _{2}\left(1+\operatorname{SNR}_{i}[t]\right)
$$

Moreover, due to renewable energy usage at the BS, the total transmit power in each time slot is subject to both the energy causality constraint and the battery storage constraint. The energy causality constraint models the fact that energy cannot be used before it arrives, and the battery storage constraint takes into consideration the fact that the finite battery storage capacity (which is denoted by $B_{\max }$ ). Let $\epsilon[t]$ be the amount of energy that can be harvested by the BS at the beginning of time slot $t^{1}$. Since the battery capacity is assumed to be finite, the energy arrival $\epsilon[t]$ may not be stored completely into the battery. To model this effect, an additional variable $B_{\text {in }}[t]$ is introduced to represent the energy actually stored into the battery at time $t$. Notice that $B_{\text {in }}[t] \leq \epsilon[t]$, for all $t$. By defining $\Delta$ as the duration of each time slot, the energy causality and the battery storage constraints can be written as

$$
\sum_{t=1}^{\ell} \sum_{i=1}^{K}\left\|\mathbf{w}_{i}[t]\right\|^{2} \Delta \leq \sum_{t=1}^{\ell} B_{\text {in }}[t]
$$

for $\ell=1, \cdots, T$, and

$$
\sum_{t=1}^{\ell+1} B_{\text {in }}[t]-\sum_{t=1}^{\ell} \sum_{i=1}^{K}\left\|\mathbf{w}_{i}[t]\right\|^{2} \Delta \leq B_{\max }
$$

for $\ell=1, \cdots, T-1$. Without loss of generality, we set $\Delta=1$ throughout the remainder of this paper.

\footnotetext{
${ }^{1}$ Here, $\epsilon[t]$ can also be viewed as the energy arriving in the previous time slot, i.e. slot $t-1$, but is not used until time slot $t$. This assumption is reasonable since, in practice, the transmission decision can only be made based on the battery state information available at the beginning of the slot.
} 
In this work, we consider the design of ZF beamforming and power control policies based on two optimizing criteria, namely, the maximum sum-rate and the minimum energycost criteria. Offline policies are first derived for both scenarios based on non-causal knowledge of the energy arrivals $\left\{\epsilon_{i}[t], \forall i, t\right\}$ and channel vectors $\left\{\mathbf{h}_{i}[t], \forall i, t\right\}$. Then, heuristic online policies are derived following intuitions obtained from the offline solutions. Specifically, in the sum-rate maximization (SRMax) problem, the BS is assumed to be supported only by renewable energy and the goal is to maximize the sum-rate over all users subject to energy causality, battery storage, and ZF constraints. The offline SRMax problem can be formulated as follows:

\section{Sum-Rate Maximization (SRMax) Problem:}

$$
\begin{aligned}
\max _{\mathbf{w}_{i}[t], B_{\text {in }}[t], \forall i, \forall t} & \sum_{t=1}^{T} \sum_{i=1}^{K} \log _{2}\left(1+\frac{\left|\mathbf{h}_{i}[t]^{H} \mathbf{w}_{i}[t]\right|^{2}}{\sigma_{n}^{2}}\right) \\
\text { subject to } & \sum_{t=1}^{\ell} \sum_{i=1}^{K}\left\|\mathbf{w}_{i}[t]\right\|^{2} \leq \sum_{t=1}^{\ell} B_{\text {in }}[t], \\
& \sum_{t=1}^{\ell+1} B_{\text {in }}[t]-\sum_{t=1}^{\ell} \sum_{i=1}^{K}\left\|\mathbf{w}_{i}[t]\right\|^{2} \leq B_{\max }, \\
& \mathbf{h}_{i}[t]^{H} \mathbf{w}_{j}[t]=0, \quad \forall i \neq j, \\
& 0 \leq B_{\text {in }}[t] \leq \epsilon[t], \forall i, \forall \ell, \forall t .
\end{aligned}
$$

On the other hand, in the energy-cost minimization (ECMin) problem, the BS is assumed to be supported by both renewable and grid energy, and the goal is to minimize the total cost of grid energy usage subject to QoS constraints at all users as well as energy causality and battery storage constraints. Suppose that the energy consumed from the grid is not stored in the battery for later use and its cost is computed according a time-varying convex cost function $f_{t}$. In this case, the cost of consuming energy $B_{\text {grid }}[t]$ from the power grid in time slot $t$ is given by $f_{t}\left(B_{\text {grid }}[t]\right)$. Moreover, the QoS constraints at the users are represented by $\operatorname{SNR}_{i}[t] \geq \gamma_{i}$, for all $i$ and $t$, where $\gamma_{i}>0$ is the target SNR value at user $i$. Hence, the offline ECMin problem can be formulated as:

\section{Energy-Cost Minimization (ECMin) Problem:}

$$
\begin{aligned}
& \min _{\mathbf{w}_{i}[t], B_{\text {grid }}[t], B_{\text {in }}[t]} \sum_{t=1}^{T} f_{t}\left(B_{\text {grid }}[t]\right) \\
& \text { subject to } \\
& \sum_{t=1}^{\ell}\left(\sum_{i=1}^{K}\left\|\mathbf{w}_{i}[t]\right\|^{2}-B_{\text {grid }}[t]\right) \leq \sum_{t=1}^{\ell} B_{\text {in }}[t], \\
& \sum_{t=1}^{\ell+1} B_{\text {in }}[t]-\sum_{t=1}^{\ell}\left(\sum_{i=1}^{K}\left\|\mathbf{w}_{i}[t]\right\|^{2}-B_{\text {grid }}[t]\right) \leq B_{\max } \\
& \frac{\left.\mid \mathbf{h}_{i}[t]\right]\left.^{H} \mathbf{w}_{i}[t]\right|^{2}}{\sigma_{n}^{2}} \geq \gamma_{i}, \quad(5 \mathrm{~d}), \quad(5 \mathrm{e}), \\
& 0 \leq B_{\text {grid }}[t] \leq \sum_{i=1}^{K}\left\|\mathbf{w}_{i}[t]\right\|^{2}, \forall i, \forall \ell, \forall t
\end{aligned}
$$

The following statement can be made for both the SRMax and the ECMin problems.

Lemma 1: The choice of renewable energy storage

$$
B_{\text {in }}^{*}[t]=\min \left\{\epsilon[t], B_{\max }\right\}, \text { for } t=1, \ldots, T,
$$

is optimal for both the SRMax and the ECMin problems.

Proof: This has been proved for the SRMax problem in our prior work [20]. To show this for the ECMin problem, let us take $\left\{\mathbf{w}_{i}^{*}[t], B_{\text {grid }}^{*}[t], B_{\text {in }}^{*}[t], \forall i, \forall t\right\}$ as the optimal solution for the problem in (6). In this case, the total energy consumption (including both renewable and grid energy) is given by $\sum_{t=1}^{T} \sum_{i=1}^{K}\left\|\mathbf{w}_{i}^{*}[t]\right\|^{2}$ and the minimum energy-cost is $\sum_{t=1}^{T} f_{t}\left(B_{\text {grid }}^{*}[t]\right)$. Suppose that there exists $t^{\prime}$ such that $B_{\text {in }}^{*}\left[t^{\prime}\right]<\min \left\{\epsilon\left[t^{\prime}\right], B_{\max }\right\}$. If the battery storage constraint is not active at time $t^{\prime}$, then there would be no loss of optimality by increasing $B_{\text {in }}^{*}\left[t^{\prime}\right]$ until it equals $\epsilon[t]$ or until the battery storage reaches $B_{\max }$. If $B_{\text {in }}^{*}\left[t^{\prime}\right]<B_{\max }$ in the latter case, then more energy can be consumed in previous time slots so that no energy is carried over to time $t^{\prime}$ and, thus, allowing $B_{\text {in }}^{*}\left[t^{\prime}\right]$ to be equal to $B_{\max }$. If this can be done by expending more renewable energy in previous time slots, then no optimality is lost. However, if this must be done by reducing the amount of grid energy consumed in previous time slots, then a lower energy cost can be achieved, which contradicts the assumption that $\left\{\mathbf{w}_{i}^{*}[t], B_{\text {grid }}^{*}[t], B_{\text {in }}^{*}[t], \forall i, \forall t\right\}$ is optimal.

This lemma shows that the renewable energy stored into the battery at each time instant should be equal to the energy arrival at the beginning of slot $t$ if the arrival does not exceed $B_{\max }$, i.e., $\epsilon[t]<B_{\max }$, and should be equal to $B_{\max }$, otherwise. This implies that, if the remaining battery capacity is not sufficient for the storage of the energy arrival in the current time slot, then more renewable energy should be consumed in previous time slots to make room for the current energy arrival. Based on this observation, $B_{\text {in }}[t]$ can be replaced with $\min \left\{\epsilon[t], B_{\max }\right\}$ without loss of optimality.

Notice that the problems in (5) and (6) are non-convex in their original forms. However, we show in the following sections that these problems can be transformed into convex optimization problems and can be solved efficiently using offthe-shelf solvers, such as CVX [24].

\section{OFFline ZERO-ForCING BEAMFORMING AND Power CONTROL POLICIES FOR SUM-RATE MAXIMIZATION}

In this section, offline ZF beamforming and power control policies are derived with the goal of maximizing the sum rate over all users and time instants.

Specifically, let us define $\mathbf{Q}_{i}[t]=\mathbf{w}_{i}[t] \mathbf{w}_{i}^{H}[t]$ as the transmit covariance matrix corresponding to the signal for user $i$. Notice that $\mathbf{Q}_{i}[t]$ is a positive semidefinite matrix with rank equal to 1 . In this case, the $\mathrm{ZF}$ constraint in $(5 \mathrm{~d})$ can be equivalently represented as $\mathbf{h}_{i}^{H}[t] \mathbf{Q}_{j}[t] \mathbf{h}_{i}[t]=0$, for all $i \neq j$. By substituting $B_{\text {in }}[t]$ with its optimal value $B_{\text {in }}[t]^{*}$, as given 
in (7), the SRMax problem in (5) can be reformulated as:

$$
\begin{array}{ll}
\max _{\mathbf{Q}_{i}[t], \forall i, \forall t} & \sum_{t=1}^{T} \sum_{i=1}^{K} \log _{2}\left(1+\frac{\mathbf{h}_{i}^{H}[t] \mathbf{Q}_{i}[t] \mathbf{h}_{i}[t]}{\sigma_{n}^{2}}\right) \\
\text { subject to } & \sum_{t=1}^{\ell} \sum_{i=1}^{K} \operatorname{tr}\left(\mathbf{Q}_{i}[t]\right) \leq \sum_{t=1}^{\ell} B_{\text {in }}^{*}[t], \\
& \sum_{t=1}^{\ell+1} B_{\mathrm{in}}^{*}[t]-\sum_{t=1}^{\ell} \sum_{i=1}^{K} \operatorname{tr}\left(\mathbf{Q}_{i}[t]\right) \leq B_{\max }, \\
& \mathbf{Q}_{i}[t] \succeq 0, \operatorname{rank}\left(\mathbf{Q}_{i}[t]\right)=1, \forall i, \forall \ell, \forall t, \\
& \mathbf{h}_{i}^{H}[t] \mathbf{Q}_{j}[t] \mathbf{h}_{i}[t]=0, \forall i \neq j .
\end{array}
$$

Notice that this problem is non-convex due to the rank-1 and $\mathrm{ZF}$ constraints in (8d) and (8e). However, following the approach given in [18], the optimal structure of $\mathbf{Q}_{i}[t]$ can be derived explicitly, which allows for the optimal decomposition of the problem in (8) into the optimization of the beamforming direction and that of the transmit power.

Specifically, let

$$
\mathbf{G}_{i}[t] \triangleq\left[\mathbf{h}_{1}[t], \mathbf{h}_{2}[t], \cdots, \mathbf{h}_{i-1}[t], \mathbf{h}_{i+1}[t], \cdots, \mathbf{h}_{K}[t]\right]^{H}
$$

be a $K-1 \times M$ matrix whose rows are the conjugate transpose of the channel vectors of all users other than user $i$. In this case, the constraints in (8e) can be expressed as

$$
\mathbf{G}_{i}[t] \mathbf{Q}_{i}[t] \mathbf{G}_{i}[t]^{H}=\mathbf{0} \text {, for all } i .
$$

Let the (reduced) singular value decomposition of $\mathbf{G}_{i}[t]$ be denoted as $\mathbf{G}_{i}[t]=\mathbf{U}_{i}[t] \Sigma_{i}[t] \mathbf{V}_{i}^{H}[t]$, where $\mathbf{U}_{i}[t] \in \mathbb{C}^{K-1 \times K-1}$ is unitary (i.e., $\mathbf{U}_{i}[t] \mathbf{U}_{i}[t]^{H}=\mathbf{U}_{i}[t]^{H} \mathbf{U}_{i}[t]=\mathbf{I}$ ), $\mathbf{V}_{i}[t] \in$ $\mathbb{C}^{M \times K-1}$ is semi-unitary (i.e., $\mathbf{V}_{i}[t]^{H} \mathbf{V}_{i}[t]=\mathbf{I}$ ), and $\Sigma_{i}[t]$ is an $K-1 \times K-1$ diagonal matrix of singular values. Moreover, let us define the orthogonal complement projection matrix $\mathbf{P}_{i}[t]=\left(\mathbf{I}-\mathbf{V}_{i}[t] \mathbf{V}_{i}^{H}[t]\right)$. Without loss of generality, the matrix $\mathbf{P}_{i}[t]$ can be expressed as $\mathbf{P}_{i}[t]=\tilde{\mathbf{V}}_{i}[t] \tilde{\mathbf{V}}_{i}^{H}[t]$, where $\tilde{\mathbf{V}}_{i}[t] \in \mathbb{C}^{M \times(M-K+1)}$ satisfies $\mathbf{V}_{i}^{H}[t] \tilde{\mathbf{V}}_{i}[t]=\mathbf{0}$ and $\tilde{\mathbf{V}}_{i}^{H}[t] \tilde{\mathbf{V}}_{i}[t]=\mathbf{I}$. The optimal structure of $\mathbf{Q}_{i}[t]$ can then be obtained explicitly as shown in [18] and summarized in the following lemma.

Lemma 2 ([18]): The ZF constraint yields the optimal structure of $\mathbf{Q}_{i}[t]$ as

$$
\mathbf{Q}_{i}[t]=\tilde{\mathbf{V}}_{i}[t] \tilde{\mathbf{Q}}_{i}[t] \tilde{\mathbf{V}}_{i}^{H}[t], \forall i, t,
$$

where $\tilde{\mathbf{Q}}_{i}[t]$ is a $(M-K+1)$-by- $(M-K+1)$ positive semidefinite matrix.

Notice that the ZF constraints in (9) (and, thus, (8e)) are satisfied with the choice of $\mathbf{Q}_{i}[t]$ in the above lemma. It is also easy to verify that $\operatorname{tr}\left(\mathbf{Q}_{i}[t]\right)=\operatorname{tr}\left(\tilde{\mathbf{Q}}_{i}[t]\right)$ and, thus, the power constraint on $\mathbf{Q}_{i}[t]$ in problem (8) can be replaced with that on $\tilde{\mathbf{Q}}_{i}[t]$. Moreover, since $\mathbf{Q}_{i}[t]$ has rank 1 and $\tilde{\mathbf{V}}_{i}[t]$ has rank $M-K+1 \geq 1, \tilde{\mathbf{Q}}_{i}[t]$ must also have rank 1 and, thus, can be further expressed as $\tilde{\mathbf{Q}}_{i}[t]=\tilde{\mathbf{q}}_{i}[t] \tilde{\lambda}_{i}[t] \tilde{\mathbf{q}}_{i}[t]^{H}$, where $\tilde{\mathbf{q}}_{i}[t]$ is a unit norm vector and $\tilde{\lambda}_{i}[t]$ is the power of $\tilde{\mathbf{Q}}_{i}[t]$. By substituting the optimal structure of $\mathbf{Q}_{i}[t]$ into (8), the problem can then be rewritten as:

$$
\begin{aligned}
\max _{\tilde{\lambda}_{i}[t], \tilde{\mathbf{q}}_{i}[t], \forall i, t} & \sum_{t=1}^{T} \sum_{i=1}^{K} \log _{2}\left(1+\frac{\tilde{\lambda}_{i}[t]\left|\mathbf{h}_{i}^{H}[t] \tilde{\mathbf{V}}_{i}[t] \tilde{\mathbf{q}}_{i}[t]\right|^{2}}{\sigma_{n}^{2}}\right) \\
\text { subject to } & \sum_{t=1}^{\ell} \sum_{i=1}^{K} \tilde{\lambda}_{i}[t] \leq \sum_{t=1}^{\ell} B_{\text {in }}^{*}[t], \\
& \sum_{t=1}^{\ell+1} B_{\text {in }}^{*}[t]-\sum_{t=1}^{\ell} \sum_{i=1}^{K} \tilde{\lambda}_{i}[t] \leq B_{\max }, \\
& \left\|\tilde{\mathbf{q}}_{i}[t]\right\|=1, \quad \tilde{\lambda}_{i}[t] \geq 0, \forall i, \forall \ell, \forall t .
\end{aligned}
$$

Notice that the choice of $\tilde{\mathbf{q}}_{i}[t]$ is independent of the choice of $\left\{\tilde{\lambda}_{i}[t], \forall i, t\right\}$ and is also independent of the choice of $\tilde{\mathbf{q}}_{j}[t]$, for all $j \neq i$. In fact, the optimal $\tilde{\mathbf{q}}_{i}[t]$ can be found by maximizing the rate of the corresponding user and time instant. The problem can be formulated as:

$$
\begin{aligned}
\max _{\tilde{\mathbf{q}}_{i}[t]} & \left|\mathbf{h}_{i}^{H}[t] \tilde{\mathbf{V}}_{i}[t] \tilde{\mathbf{q}}_{i}[t]\right|^{2} \\
\text { subject to } & \left\|\tilde{\mathbf{q}}_{i}[t]\right\|=1 .
\end{aligned}
$$

The optimal $\tilde{\mathbf{q}}_{i}[t]$ is thus given by

$$
\tilde{\mathbf{q}}_{i}^{*}[t]=\tilde{\mathbf{V}}_{i}[t]^{H} \mathbf{h}_{i}[t] /\left\|\tilde{\mathbf{V}}_{i}[t]^{H} \mathbf{h}_{i}[t]\right\| .
$$

By substituting $\tilde{\mathbf{q}}_{i}^{*}[t]$ into (10), the optimal transmit covariance matrix can be written as $\mathbf{Q}_{i}^{*}[t]=\tilde{\mathbf{V}}_{i}[t] \tilde{\mathbf{q}}_{i}^{*}[t] \lambda_{i}[t] \tilde{\mathbf{q}}_{i}^{*}[t]^{H} \tilde{\mathbf{V}}_{i}[t]^{H}$ and, thus, the optimal $\mathrm{ZF}$ beamforming vector for user $i$ is $\mathbf{w}_{i}^{*}[t]=\sqrt{\tilde{\lambda}_{i}[t]} \tilde{\mathbf{V}}_{i}[t] \tilde{\mathbf{V}}_{i}[t]^{H} \mathbf{h}_{i}[t] /\left\|\tilde{\mathbf{V}}_{i}[t]^{H} \mathbf{h}_{i}[t]\right\|$. That is, the optimal ZF beamforming direction is given by the projection of $\mathbf{h}_{i}[t]$ onto the null space of all other users' channels.

Given the ZF beamforming vectors $\left\{\mathbf{w}_{i}^{*}[t]\right\}$, the remaining power control problem can then be formulated as

$$
\begin{array}{ll}
\max _{\lambda_{i}[t], \forall i, \forall t} & \sum_{t=1}^{T} \sum_{i=1}^{K} \log _{2}\left(1+\frac{\left.\lambda_{i}[t] \| \tilde{\mathbf{V}}_{i}[t]\right]^{H} \mathbf{h}_{i}[t] \|^{2}}{\sigma_{n}^{2}}\right) \\
\text { subject to } & \sum_{t=1}^{\ell} \sum_{i=1}^{K} \lambda_{i}[t] \leq \sum_{t=1}^{\ell} B_{\text {in }}^{*}[t], \\
& \sum_{t=1}^{\ell+1} B_{\text {in }}^{*}[t]-\sum_{t=1}^{\ell} \sum_{i=1}^{K} \lambda_{i}[t] \leq B_{\max }, \\
& \lambda_{i}[t] \geq 0, \quad \forall i, \forall \ell, \forall t .
\end{array}
$$

The problem is convex and can be solved by considering its Lagrangian dual problem and by applying the KKT optimality conditions [25]. This problem can be solved following the approach in [20] and leads to a so-called directional waterfilling algorithm [19]. The algorithm is summarized as follows:

\section{Directional Water-filling Algorithm: \\ Initialize:}

Initial water level $1 / \tau_{\ell}^{(0)}$ by traditional water-filling algorithm with total power consumption at slot $\ell$ is $B_{\text {in }}^{*}[\ell]$.

\section{Update Procedure of $n$-th Iteration:}

(i) Starting from first time slot, compare the water level for consecutive time slots $\ell$ and $\ell+1$. 
(ii) If $1 / \tau_{\ell}^{(n-1)} \leq 1 / \tau_{\ell+1}^{(n-1)}$, then set $1 / \tau_{\ell}^{(n)}=1 / \tau_{\ell}^{(n-1)}$ and jump to the next slot. Else, goto step (iii).

(iii) Let $1 / \tau_{\ell}^{(n)}=1 / \tau_{\ell+1}^{(n)}$ by performing waterfilling over the two time slots and check whether or not the battery storage constraint is satisfied. If the constraint is satisfied, then jump to next slot. Else, goto step (iv).

(iv) Set the total transmit energy in slot $\ell+1$ as $B_{\max }$, and recalculate the available energy in slot $\ell$ as well as the waterlevels $1 / \tau_{\ell}^{(n)}$ and $1 / \tau_{\ell+1}^{(n)}$.

(v) Repeat (ii)-(iv) until $\ell=T-1$.

(vi) Stop if $\left|1 / \tau_{\ell}^{(n-1)}-1 / \tau_{\ell}^{(n)}\right| \leq \delta$, for all $\ell$. Else, let $n=$ $n+1$ and goto (i).

The directional water-filling algorithm ensures that the water-level is monotonically increasing unless the battery storage constraint is active in a certain time slot. Please see [19] and [20] for further details.

\section{OfFline ZERo-Forcing BeAmForming AND Power Control Policies For EnERGY-COST Minimization}

In this section, offline ZF beamforming and power control policies are derived with the goal of minimizing the cost of using grid energy when renewable energy is not sufficient to guarantee QoS at all users. Similar to Section III, let $\mathbf{Q}_{i}[t]=$ $\mathbf{w}_{i}[t] \mathbf{w}_{i}[t]^{H}$ be the transmit covariance matrix corresponding to the signal for user $i$. By expressing the ZF constraint as $\mathbf{h}_{i}^{H}[t] \mathbf{Q}_{j}[t] \mathbf{h}_{i}[t]=0$, for all $i \neq j$, the ECMin problem in (6) can be formulated as:

$$
\min _{\mathbf{Q}_{i}[t], B_{\text {grid }}[t], B_{\text {in }}[t], \forall i, t} \sum_{t=1}^{T} f_{t}\left(B_{\text {grid }}[t]\right)
$$

subject to

$$
\begin{aligned}
& \sum_{t=1}^{\ell}\left(\sum_{i=1}^{K} \operatorname{tr}\left(\mathbf{Q}_{i}[t]\right)-B_{\text {grid }}[t]\right) \leq \sum_{t=1}^{\ell} B_{\text {in }}[t] \\
& \sum_{t=1}^{\ell+1} B_{\text {in }}[t]-\sum_{t=1}^{\ell}\left(\sum_{i=1}^{K} \operatorname{tr}\left(\mathbf{Q}_{i}[t]\right)-B_{\text {grid }}[t]\right) \leq B_{\text {max }}, \\
& \mathbf{h}_{i}^{H}[t] \mathbf{Q}_{i}[t] \mathbf{h}_{i}[t] / \sigma_{n}^{2} \geq \gamma_{i}, \quad(8 \mathrm{~d}), \quad(8 \mathrm{e}), \\
& 0 \leq B_{\text {grid }}[t] \leq \sum_{i=1}^{K} \operatorname{tr}\left(\mathbf{Q}_{i}[t]\right) \\
& 0 \leq B_{\text {in }}[t] \leq \epsilon[t], \forall i, \forall \ell, \forall t
\end{aligned}
$$

Notice from the above formulation that, different from the SRMax problem, we do not choose to replace $B_{\text {in }}[t]$ with $B_{\text {in }}^{*}[t]$ given in Lemma 1, even though it has been shown that there is no loss of optimality in doing so. However, this does require the BS to consume more power than needed in earlier time slots to save room in the battery for energy storage in later time slots (c.f., proof of Lemma 1). This would be inefficient in the online scenario, to be discussed in the next section, where future energy arrival is not known a priori. Hence, to make this problem extendable to the online scenario, we leave $B_{\text {in }}[t]$ as a design parameter here.

Similarly, by Lemma (2), let us write the optimal transmit covariance as $\mathbf{Q}_{i}[t]=\tilde{\mathbf{V}}_{i}[t] \tilde{\mathbf{Q}}_{i}[t] \tilde{\mathbf{V}}_{i}^{H}[t]$, where $\tilde{\mathbf{V}}_{i}[t]$ is as defined before and $\tilde{\mathbf{Q}}_{i}[t]$ is a positive semidefinite matrix that can be decomposed as $\tilde{\mathbf{q}}_{i}[t] \tilde{\lambda}_{i}[t] \tilde{\mathbf{q}}_{i}^{H}[t]$, where $\tilde{\lambda}_{i}[t]$ is the power of $\mathbf{Q}_{i}[t]$ and $\tilde{\mathbf{q}}_{i}[t]$ is a unit norm vector. The problem (16) can be reformulated as:

$\min _{\tilde{\mathbf{q}}_{i}[t], \tilde{\lambda}_{i}[t], B_{\text {grid }}[t], B_{\text {in }}[t], \forall i, t} \sum_{t=1}^{T} f_{t}\left(B_{\text {grid }}[t]\right)$

subject to

$$
\begin{aligned}
& \frac{\tilde{\lambda}_{i}[t]\left|\mathbf{h}_{i}^{H}[t] \tilde{\mathbf{V}}_{i}[t] \tilde{\mathbf{q}}_{i}[t]\right|^{2}}{\sigma_{n}^{2}} \geq \gamma_{i} \\
& \sum_{t=1}^{\ell}\left(\sum_{i=1}^{K} \tilde{\lambda}_{i}[t]-B_{\text {grid }}[t]\right) \leq \sum_{t=1}^{\ell} B_{\text {in }}[t] \\
& \sum_{t=1}^{\ell+1} B_{\text {in }}[t]-\sum_{t=1}^{\ell}\left(\sum_{i=1}^{K} \tilde{\lambda}_{i}[t]-B_{\text {grid }}[t]\right) \leq B_{\max }, \\
& 0 \leq B_{\text {grid }}[t] \leq \sum_{k=1}^{K} \tilde{\lambda}_{i}[t] \\
& 0 \leq B_{\text {in }}[t] \leq \epsilon[t], \forall i, \forall \ell, \forall t
\end{aligned}
$$

Notice that $\tilde{\mathbf{q}}_{i}[t]$ appears only in the QoS constraint. To minimize the energy cost, one should choose $\tilde{\mathbf{q}}_{i}[t]$ such that the SNR of user $i$ is as large as possible. As a result, the energy required to achieve the SNR constraint can be minimized. Hence, the search for the optimal $\tilde{\mathbf{q}}_{i}[t]$ can also be formulated as in (12) and can be found separately for each user. The optimal value of $\tilde{\mathbf{q}}_{i}[t]$ is also given by (14). Given that $\tilde{\mathbf{q}}_{i}^{*}[t]$ is chosen, the optimal value of $\lambda_{i}[t]$ should be chosen such that the SNR constraint is met with equality, which is given by $\tilde{\lambda}_{i}^{*}[t]=\sigma_{n}^{2} \gamma_{i} /\left|\mathbf{h}_{i}^{H}[t] \tilde{\mathbf{V}}_{i}[t] \tilde{\mathbf{q}}_{i}^{*}[t]\right|^{2}$. The offline ECMin problem reduces to the following optimization problem:

$$
\min _{B_{\text {grid }}[t], B_{\text {in }}[t]} \sum_{t=1}^{T} f_{t}\left(B_{\text {grid }}[t]\right)
$$

subject to

$$
\begin{aligned}
& \sum_{t=1}^{\ell}\left(\sum_{i=1}^{K} \tilde{\lambda}_{i}^{*}[t]-B_{\text {grid }}[t]\right) \leq \sum_{t=1}^{\ell} B_{\text {in }}[t] \\
& \sum_{t=1}^{\ell+1} B_{\text {in }}[t]-\sum_{t=1}^{\ell}\left(\sum_{i=1}^{K} \tilde{\lambda}_{i}^{*}[t]-B_{\text {grid }}[t]\right) \leq B_{\max }, \\
& 0 \leq B_{\text {grid }}[t] \leq \sum_{k=1}^{K} \tilde{\lambda}_{i}^{*}[t] \\
& 0 \leq B_{\text {in }}[t] \leq \epsilon[t], \forall \ell, \forall t .
\end{aligned}
$$

The above problem is a convex optimization problem which can be solved efficiently using general purpose interior point solvers such as CVX [24]. The ECMin problem will be evaluated in Section VI for quadratic costs, i.e., $f_{t}\left(B_{\text {grid }}[t]\right)=$ $\alpha_{t}\left(B_{\text {grid }}[t]\right)^{2}$.

It is worthwhile to remark that, by considering a convex cost function, the BS is penalized more for consuming a large amount of grid energy at any given time. Hence, it is more desirable to spread the grid energy consumption over time. 
Even though we assume that grid energy is not stored into the battery, this can still be achieved by reallocating the renewable energy usage over time. Moreover, when the cost function varies over time, it is also desirable to utilize less renewable energy (when the grid energy cost is low), and leave it for use in later time slots (when grid energy cost is high).

\section{EXTENSIONS TO ON-LINE ZERO-FORCING BEAMFORMing AND POWER CONTROL POLICIES}

In this section, online policies are proposed to mimic the energy flow in the offline policies derived in previous sections.

Recall that, in the previous sections, the ZF beamforming vector can be found independently for each user and time slot, regardless of the assigned power values, and the solution is given by

$$
\mathbf{w}_{i}^{*}[t]=\sqrt{\tilde{\lambda}_{i}[t]} \tilde{\mathbf{V}}_{i}[t] \tilde{\mathbf{V}}_{i}[t]^{H} \mathbf{h}_{i}[t] /\left\|\tilde{\mathbf{V}}_{i}[t]^{H} \mathbf{h}_{i}[t]\right\| .
$$

Hence, the parameters that remain to be determined in the SRMax problem are $\lambda_{i}[t]$, for all $i$ and $t$, whereas those in the ECMin problem are $B_{\text {grid }}[t]$, for all $t$, since the optimal values of $\lambda_{i}[t]$, for all $i$ and $t$, can be determined in this case according to the QoS constraints.

\section{A. Online Policy for Sum-Rate Maximization}

From the discussions in Section III and the directional waterfilling algorithm [19], [20], we know that the sum rate is maximized by keeping the water-level in consecutive time slots as equal as possible (or as close as possible subject to the battery storage constraint, which prevents water flowing from one slot to the next).

Let us define

$$
C\left[t^{\prime}\right]=\sum_{t=1}^{t^{\prime}} B_{\text {in }}[t]-\sum_{t=1}^{t^{\prime}-1} \sum_{i=1}^{K} \lambda_{i}[t]
$$

as the available battery energy for use in time slot $t^{\prime}$. Moreover, let $\beta_{i}\left[t^{\prime}\right]=\left|\mathbf{h}_{i}^{H}\left[t^{\prime}\right] \tilde{\mathbf{V}}_{i}\left[t^{\prime}\right] \tilde{\mathbf{q}}_{i}^{*}\left[t^{\prime}\right]\right|^{2} / \sigma_{n}^{2}$ be the effective channel gain of user $i$ in time slot $t^{\prime}$. Following intuitions from the water-filling algorithm, a certain portion of the energy available in slot $t^{\prime}$ should be stored for use in later time slots if the current water-level is higher than the (expected) waterlevel in later time slots. Let $\delta\left[t^{\prime}\right]$ be the amount of energy that should be saved in slot $t^{\prime}$. Then, our goal is to choose $\delta\left[t^{\prime}\right]$ such that

$$
\sum_{i=1}^{K}\left(\tau-\frac{1}{\beta_{i}\left[t^{\prime}\right]}\right)=C\left[t^{\prime}\right]-\delta\left[t^{\prime}\right]
$$

and

$$
\sum_{t=t^{\prime}+1}^{T} \mathrm{E}\left[\left(\tau-\frac{1}{\beta_{i}[t]}\right)^{+}\right]=\delta\left[t^{\prime}\right]+\sum_{t=t^{\prime}+1}^{T} \mathrm{E}\left[B_{\text {in }}^{*}[t]\right]
$$

for some $\tau$. Here, $\tau$ is the common water-level that is desired. Combining the above two equations and by assuming that the channel coefficients and energy arrivals are i.i.d., the problem is equivalent to finding the water-level $\tau$ such that

$$
\begin{aligned}
& \sum_{i=1}^{K}\left(\tau-\frac{1}{\beta_{i}\left[t^{\prime}\right]}\right)+\left(T-t^{\prime}+1\right) \mathrm{E}\left[\left(\tau-\frac{1}{\beta_{i}[t]}\right)^{+}\right] \\
& =C\left[t^{\prime}\right]+\left(T-t^{\prime}\right) \mathrm{E}\left[B_{\text {in }}^{*}[t]\right] .
\end{aligned}
$$

However, if the available energy $C\left[t^{\prime}\right]$ is not sufficient to achieve $\tau$ in the current time slot, the water-level is simply set as the highest level possible, i.e., the available battery energy is depleted in slot $t^{\prime}$.

\section{B. Online Policy for Energy-Cost Minimization}

For the ECMin problem, let us consider an online policy where the grid energy consumed at time $t^{\prime}$, i.e., $B_{\text {grid }}\left[t^{\prime}\right]$, is chosen to minimize the future energy-cost subject to average energy constraints. The online ECMin problem is formulated as follows:

$$
\begin{aligned}
& \min _{B_{\text {grid }}[t], B_{\text {in }}[t] \forall t \geq t^{\prime}} \sum_{t=t^{\prime}}^{T} f_{t}\left(B_{\text {grid }}[t]\right) \\
& \text { subject to } \\
& \quad \sum_{t=1}^{\ell}\left(\sum_{i=1}^{K} \overline{\tilde{\lambda}}^{*}{ }_{i}[t]-B_{\text {grid }}[t]\right) \leq \sum_{t=1}^{\ell} B_{\text {in }}[t] \\
& \ell+1 \\
& \sum_{t=1}^{\ell+1} B_{\text {in }}[t]-\sum_{t=1}^{\ell}\left(\sum_{i=1}^{K} \overline{\tilde{\lambda}^{*}}{ }_{i}[t]-B_{\text {grid }}[t]\right) \leq B_{\max } \\
& 0 \leq B_{\text {grid }}[t] \leq \sum_{k=1}^{K} \overline{\tilde{\lambda}_{i}^{*}}[t], \\
& 0 \leq B_{\text {in }}[t] \leq \mathrm{E}[\epsilon[t]], \forall i, \forall \ell \geq t^{\prime}, \forall t \geq t^{\prime}
\end{aligned}
$$

where $B_{\text {in }}[t]$, for $t \leq t^{\prime}$ is the amount of renewable energy actually stored into the battery at time $t$ and is given by $B_{\text {in }}[t]=\min \left\{\epsilon[t], B_{\max }-\sum_{s=1}^{t-1} B_{\text {in }}[s]+\right.$ $\left.\sum_{s=1}^{t-1}\left(\sum_{i=1}^{K} \tilde{\lambda}_{i}^{*}[s]-B_{\text {grid }}[s]\right)\right\}$. Here, we set $\tilde{\tilde{\lambda}}^{*}[t]=\tilde{\lambda}_{i}^{*}[t]$, for $t \leq t^{\prime}$ and $\tilde{\tilde{\lambda}}^{*}{ }_{i}[t]=\mathrm{E}\left[\tilde{\lambda}_{i}^{*}[t]\right]$, for $t>t^{\prime}$. Notice that $\overline{\tilde{\lambda}}^{*}{ }_{i}[t]$ represents its actual value when $t \leq t^{\prime}$ and is its expected value when $t>t^{\prime}$. Regardless, these values are deterministic and the problem becomes similar to its original problem in (18). That is, the problem is convex and can be solved efficiently using general purpose interior point solvers such as CVX [24]. This problem is solved for each time slot, say slot $t^{\prime}$, and yields a solution of $B_{\text {grid }}[t]$, for all $t \geq t^{\prime}$. However, only the value $B_{\text {grid }}\left[t^{\prime}\right]$ is extracted from the solution of the problem at time $t^{\prime}$ and is used to determine the grid energy that should be purchased at this time.

\section{NUMERICAL COMPARISON}

In this section, we compare the performance of the offline and online policies for both SRMax and ECMin problems. The channel fading and energy arrival are randomly generated, and the results of the experiments are obtained by averaging over 5000 realizations. The elements in the channel vectors are assumed to be i.i.d. Gaussian distribution with zero mean 


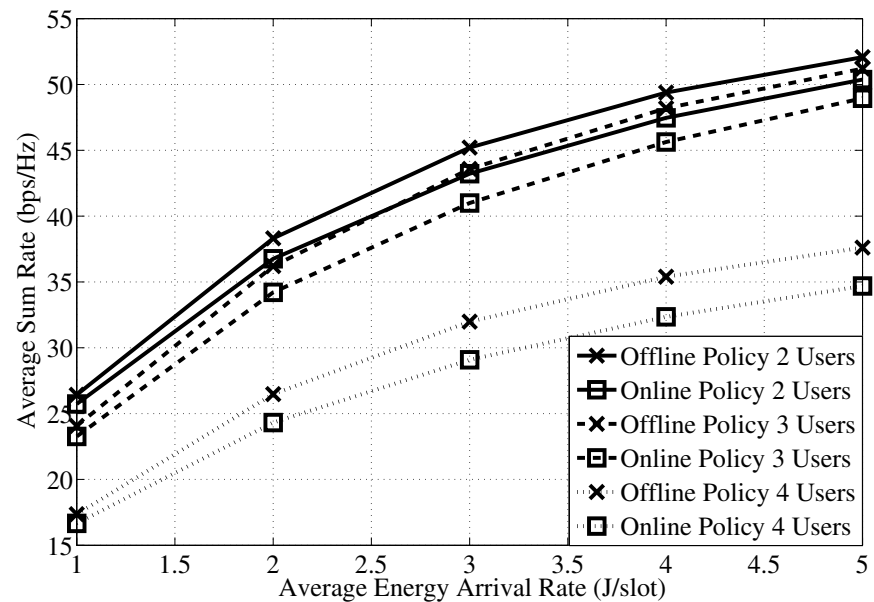

Fig. 2. Average sum rate of the policies for different energy arrival rates and different user numbers, $T=15$ and $B_{\max }=5 \mathrm{~J}$.

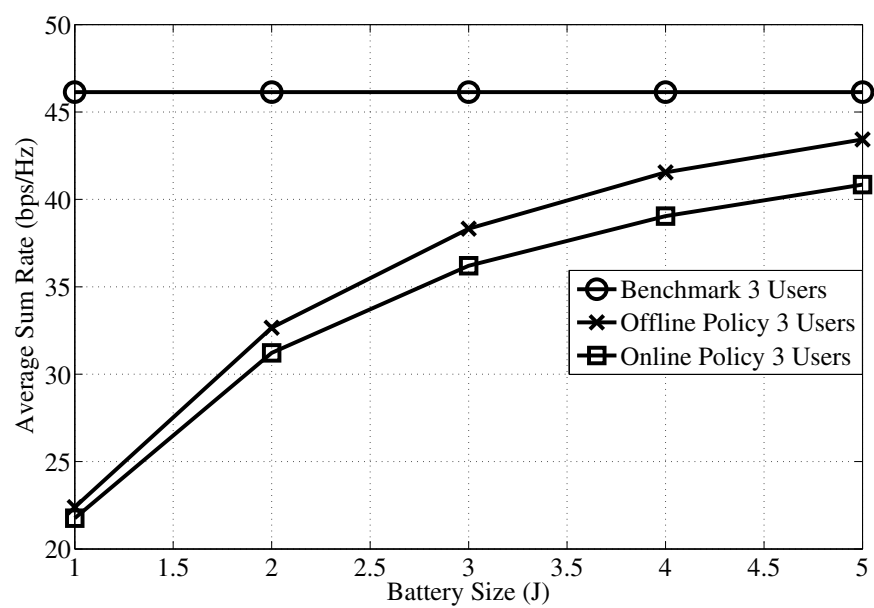

Fig. 3. Average sum rate of the policies for different battery size, $T=15$, $K=3$ and $\bar{\epsilon}=3 \mathrm{~J} /$ slot.

and variance $\sigma_{h}^{2}=1$, and the noise variance is $\sigma_{n}^{2}=1$. The number of antennas at the $\mathrm{BS}$ is $M=4$ and the deadline is $T=15$. The energy arrival in each time slot is assumed to be Gamma distributed, i.e., $\epsilon[t] \sim \mathcal{G}(\alpha, \theta)$, since it can be used to approximate many positive continuous random variables [26]. We set $\alpha=2$ and scale $\theta$ to obtain different average energy arrival rates, i.e., $\bar{\epsilon}=\alpha \theta \mathrm{J} /$ slot.

In Figs. 2, 3, and 4, the average sum rate is shown for both offline and online policies with respect to varying energy arrival, battery size, and deadline constraints. We can observe that offline policies perform better as expected, but the proposed online policy also performs reasonably close to the optimal offline policy. In Fig. 2, the sum-rate performance increases with the average energy arrival rate but decreases as the number of users increase due to the ZF constraint and, thus, the reduced design flexibility. The water-level for the online sum rate problem $\tau$ can be solved from the equation (20). Here, the expected values are computed numerically using 10000 channel realizations, and the optimal value of $\tau$ is found using bisection. In Fig. 3, we show the average sum rate with respect to different battery size. The benchmark curve corresponds to the case where the battery capacity is

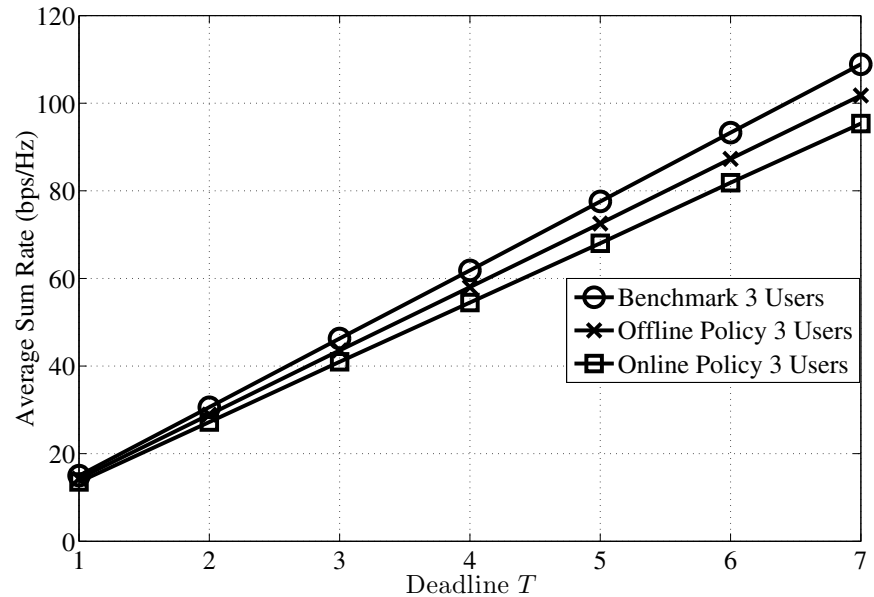

Fig. 4. Average sum rate of the policies for different transmission deadline, $K=3, \bar{\epsilon}=3 \mathrm{~J} / \mathrm{slot}$, and $B_{\max }=5 \mathrm{~J}$.

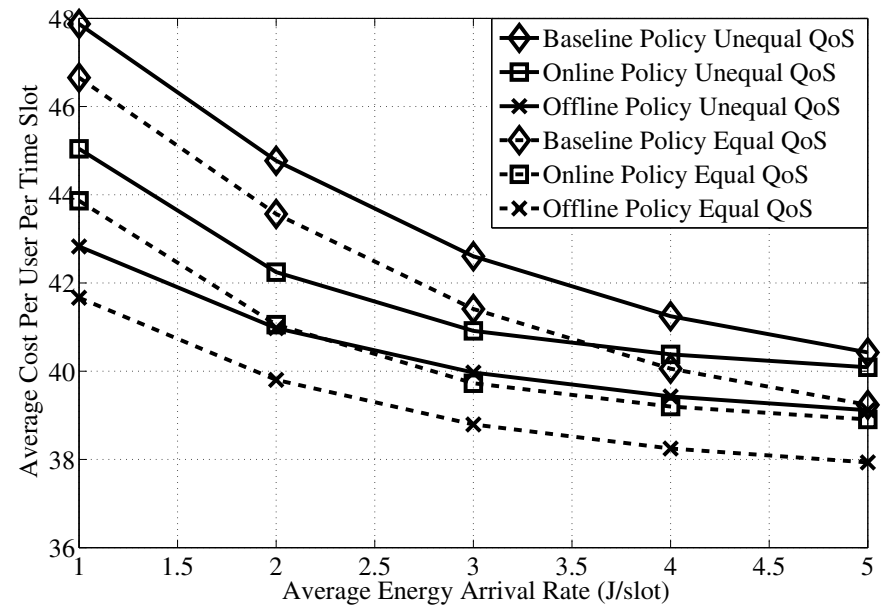

Fig. 5. Average cost of the policies for different energy arrival rates and different QoS requirement, $T=15, K=3, \bar{\epsilon}=3 \mathrm{~J} / \mathrm{slot}$, and $B_{\max }=5 \mathrm{~J}$.

infinity. We can see that the performance of both offline and online policies increases with the battery size. Moreover, in Fig. 4, we show the average sum rate of both offline and online policies under different transmission deadline. We can see that the sum rate of all policies will linearly increase in Fig. 4.

In Fig. 5, 6, and 7, we compare the average energy cost of the offline and online policies with a baseline policy where renewable energy is always used before consuming grid energy in each slot. The experiment is performed for a system with $K=3$ users. The cost function is defined as $f_{t}\left(B_{\text {grid }}[t]\right)=\alpha_{t}\left(B_{\text {grid }}[t]\right)^{2}$, where $\alpha_{t}$ is chosen randomly but fixed in all experiments. In particular, $\alpha_{t}$ is assumed to be uniform distributed within the interval $[0.5,1.5]$. The QoS requirement for the case with equal QoS is set as 2 for all users and that for the case with non-equal QoS is set as 1, 2, and 3 . We can see that the ECMin policy performs much better than the baseline strategy in Fig. 5. Also, we can observe that the energy-cost decreases monotonically as the energy arrival rate increases, but saturates when the energy arrival rate becomes comparable to the battery capacity. In this case, the renewable energy that is available for use in each time slot is limited by the battery size and, thus, no improvements can be attained 


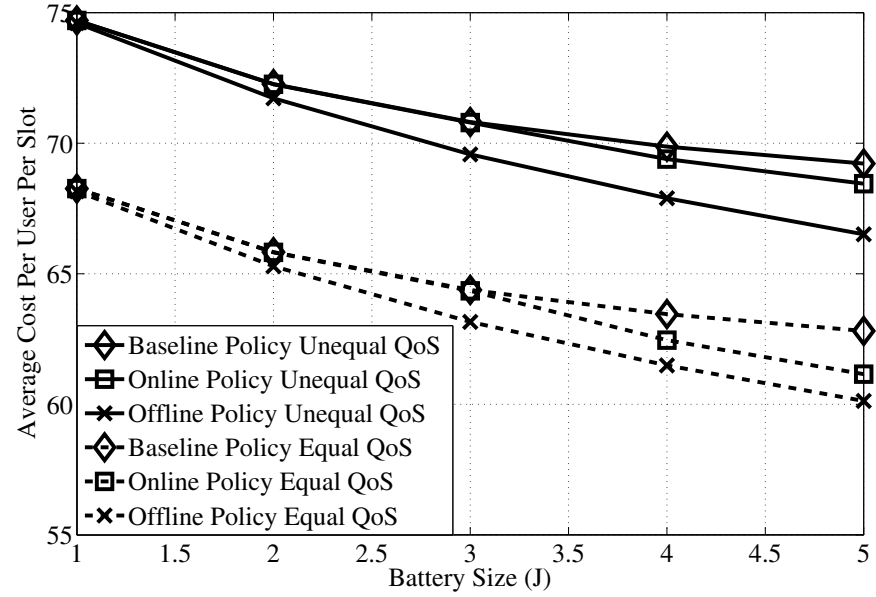

Fig. 6. Average cost of the policies for different battery size and differen QoS requirement, $T=15, K=3, \bar{\epsilon}=3 \mathrm{~J} /$ slot.

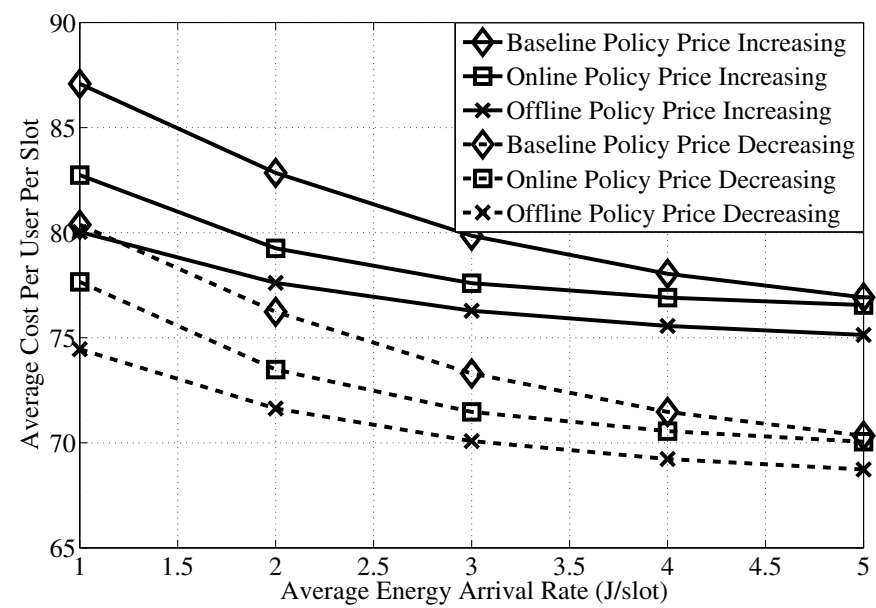

Fig. 7. Average cost of the policies for different pricing factors and different QoS requirement, $K=3, \bar{\epsilon}=3 \mathrm{~J} / \mathrm{slot}$, and $B_{\max }=5 \mathrm{~J}$.

by increasing the energy arrival rate. In Fig. 6, we show the average energy cost per user per slot with respect to varying battery size. We can see that, when the battery size is small (e.g., when $B_{\max } \leq 3 \mathrm{~J}$ ), the online policy performs close to the baseline strategy, meaning that it will tend to utilize all renewable energy in each slot. However, improvement over the baseline policy can be observed as the battery size increases.

In the above figures, $\alpha_{t}$ is assumed to be uniform distributed within the interval $[0.5,1.5]$. In Fig. 7 , we consider the case where the pricing may be non-stationary over time. Specifically, to model the case where the price increase over time, we assume that $\alpha_{t}$ is uniformly distributed between $(0.5,1.5)$ in slots $t=1 \sim 5$, between $(1,2)$ in slots $t=6 \sim 10$, and between $(1.5,2.5)$ in slots $t=11 \sim 15$. For the case where price is decreasing over time, we assume that $\alpha_{t}$ is uniformly distributed between $(1.5,2.5)$ in slots $t=1 \sim 5$, between $(1,2)$ in slots $t=6 \sim 10$, and between $(0.5,1.5)$ in slots $t=11 \sim 15$. We can see that the case with decreasing price requires less energy cost than the case with increasing cost. This is because, for the case with increasing price, the BS will tend to store more energy in earlier slots, but will be limited by the battery size when doing so.

\section{CONCLUSION}

In this work, we derived both offline and online ZF beamforming and power control policies for downlink transmission at a renewable energy empowered multi-antenna BS. Two design problems were considered, i.e., the sum-rate maximization problem and energy-cost minimization problem. In the SRMax problem, the optimal ZF beamformer can be derived explicitly and is consistent with that found in the literature for systems without renewable energy constraints. The power control problem was then shown to be convex and was shown to result in the directional water-filling algorithm. In the ECMin problem, the cost of consuming grid energy is minimized by allowing BSs to reduce the use of renewable energy in time slots with lower energy cost and store it in the battery for later use, i.e., for use in time slots with higher energy cost. Online policies were proposed for both SRMax and ECMin problems based on average future channel coefficients and energy arrivals. The effectiveness of the proposed schemes were shown through numerical simulations.

\section{REFERENCES}

[1] V. Chandrasekhar, J. Andrews, and A. Gatherer, "Femtocells Networks: A Survey," IEEE Commun. Mag., vol. 46, no. 9 pp. 59 - 67, 2008.

[2] A. Bianzino, C. Chaudet, D. Rossi, and J. Rougier, "A survey of green networking research," IEEE Commun. Surveys \& Tuts., vol. 14, no. 1, pp. $3-20,2012$

[3] C. Peel, B. Hochwald, and A. Swindlehurst, "A Vector-Perturbation Technique for Near-Capacity Multiantenna Multiuser CommunicationXPart I: Channel Inversion and Regularization," IEEE Trans. Commun., vol. 53, no. 1 , pp.195 - 202, 2005.

[4] G. Caire and S. Shamai, "On the Achievable Throughput of a Multiantenna Gaussian Broadcast Channel," IEEE Trans. Inf. Theory, vol. 49, no. 7, pp. 1691 - 1706, 2003.

[5] T. Yoo and A. Goldsmith, "On the Optimality of Multiantenna Broadcast Scheduling Using Zero-Forcing Beamforming", IEEE J. Sel. Areas Commun., vol. 24, no. 3, pp. 528 - 541, 2006.

[6] Q. H. Spencer, A. L. Swindlehurst, and M. Haardt, "Zero-forcing methods for downlink spatial multiplexing in multiuser MIMO channels," IEEE Trans. Signal Process., vol. 52, no. 2, pp. 461 - 471, 2004.

[7] J. Choi and S. Perreau, "MMSE multiuser downlink multiple antenna transmission for CDMA systems", IEEE Trans. Signal Process., vol. 52, no. 6, pp. 1564 - 1573, 2004.

[8] L.-U. Choi and R. D. Murch, "Transmit-preprocessing technique with simplified receivers for the downlink of MISO TDD-CDMA systems", IEEE Trans. Veh. Technol., vol. 53, no. 2, pp. 285 - 295, 2004.

[9] S. Serbetli and A. Yener, "Transceiver Optimization for Multiuser MIMO Systems", IEEE Trans. Signal Process., vol. 52, no. 1, 2004.

[10] E. A. Jorswieck and E. G. Larsson, "Complete characterization of pareto boundary for the MISO interference channel", IEEE Trans. Signal Process., vol. 56, no. 10, pp. 5292 - 5296, 2008.

[11] E. A. Jorswieck and E. G. Larsson, "The MISO interference channel from a game-theoretic perspective: a combination of selfishness and altruism achieves pareto optimality", in Proc. IEEE ICASSP, 2008, pp. 5364 - 5367.

[12] M. Kobayashi and G. Caire, "Joint Beamforming and Scheduling for a Multi-Antenna Downlink with Imperfect Transmitter Channel Knowledge“, IEEE J. Sel. Areas Commun., vol. 25, no. 7, pp. 1468 - 1477, 2007.

[13] W. Yang and G. Xu, "Optimal downlink power assignment for smart antenna systems", in Proc. IEEE ICASSP, 1998, vol. 6, pp. 3337 - 3340

[14] A. Wiesel, Y. Eldar, and S. Shamai, "Linear precoding via conic optimization for fixed MIMO receivers", IEEE Trans. Signal Process., vol. 54, no. 1, pp. 161 - 176, 2006.

[15] F. Negro, M. Cardone, I. Ghauri, and D. T.M. Slock, "SINR balancing and beamforming for the MISO interference channel", in Proc. IEEE PIMRC, 2011, pp. 1552 - 1556. 
[16] M. Bengtsson and B. Ottersten, "Optimal and suboptimal transmit beamforming," Chapter 18 in Handbook of Antennas in Wireless Communications. L. C. Godara, Ed., CRC Press, 2001.

[17] M. Schubert and H. Boche, "Solution of the multiuser downlink beamfwming problem with individual SINR constraints," IEEE Trans. Veh. Technol., no.1, pp. 18 - 28, 2004.

[18] R. Zhang, "Cooperative Multi-Cell Block Diagonalization with PerBase-Station Power Constraints," IEEE Journal on Selected Areas in Communications, vol. 28, no. 9, pp. 1435 - 1445, 2010.

[19] O. Ozel, K. Tutuncuoglu, J. Yang, S. Ulukus, and A. Yener, "Transmission with Energy Harvesting Nodes in Fading Wireless Channels: Optimal Policies," IEEE J. Sel. Areas Commun., vol. 29, no. 8, pp. 1732 - 1743, 2011.

[20] Y.-S. Wang, Y.-W. Peter Hong, and W.-T. Chen, "Downlink Multiuser Beamforming and Power Control for Base Stations Empowered by Renewable Energy," in Proc. IEEE PIMRC, 2013.

[21] K. Tutuncuoglu and A. Yener, "Optimum Transmission Policies for Battery Limited Energy Harvesting Nodes," IEEE Trans. Wireless Commun., vol. 11, no. 3, pp. 1180 - 1189, 2012.

[22] O. Ozel, J. Yang, and S. Ulukus, "Optimal Broadcast Scheduling for an Energy Harvesting Rechargeable Transmitter with a Finite Capacity Battery", IEEE Trans. Wireless Commun., vol. 11, no. 6, pp. 2193 - 2203, 2012.

[23] J. Gong, S. Zhou, and Z. Niu, "Optimal Power Allocation for Energy Harvesting and Power Grid Coexisting Wireless Communication Systems", IEEE Trans. Commun., vol. 61. no. 7, pp. 3040 - 3049, 2013.

[24] CVX Research, Inc. CVX: Matlab software for disciplined convex programming, version 2.0 beta. http://cvxr.com/cvx, September 2012

[25] S. Boyd and L. Vanderberghe, Convex Optimization. Cambridge University Press, 2004.

[26] S. Luo, R. Zhang, and T. J. Lim, "Optimal Save-Then-Transmit Protocol for Energy Harvesting Wireless Transmitters," IEEE Trans. Wireless Commun., vol. 12, no.3, pp. 1196 - 1207, 2013 